\title{
Relapsing Urinary Catheter Bleeding with Triple Antithrombotic Therapy in an Elderly Patient with ACS, PCI and A-fib: A Case Report
}

\author{
Michael AB Naafs* \\ Dutch Internist Endocrinologist, Netherlands
}

*Corresponding author: Michael AB Naafs, Dutch Internist Endocrinologist, Health Consultant at Naafs, International Health Consultancy, Netherlands

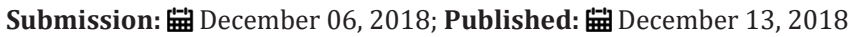

\begin{abstract}
In this case report an 89-year-old man is described with relapsing gross hematuria under new antithrombotic triple therapy for ACS, PCI and A-fib in the presence of a double-J-stent and bladder catheter, a complex therapeutic challenge. Hospital admissions of these patients already rise over 120.000 a year in the U.S.
\end{abstract}

\section{Introduction}

Antithrombotic agents are among the most commonly prescribed medications for older adults. Oral anticoagulants, including the new non-vitamin $\mathrm{K}$ antagonist oral anticoagulants (NOACs) are indicated for primary and secondary prevention of stroke and systemic embolism, as well as treatment of venous thromboembolism (VTE), atrial fibrillation (A-fib) and acute coronary syndrome (ACS). Antiplatelet agents are agents indicated for primary and secondary prevention of cardiovascular disease. In the last decade more patients, particularly elderly, use dual or triple antithrombotic therapy in cases of NSTEMI (non-ST elevation myocardial infarction), ACS and PCI (percutaneous coronary intervention). Bleeding risk is greatly increased with these new antithrombotic regimens. Published trials have focused on intracranial hemorrhage, gastrointestinal bleeding and all cause bleeding as adverse events. Gross hematuria as adverse effect of NOACs has not been examined as the primary outcome in published trials of patients treated with NOACs in combination with antiplatelet agents [1]. In this case report an elderly male patient is described with relapsing gross urinary catheter bleeding under the simultaneous use of aspirin, clopidogrel (Plavix) and apixaban (Eliquis), so called triple antithrombotic therapy.

\section{Case history}

The patient is an 89- year old frail men with an extensive atherosclerotic vascular history including femoral artery stenting for PAD, PCI, hypertension and vascular Parkinsonism complicated by multiple aspiration pneumonias. End stage kidney disease is caused by an atherosclerotic shrinkage kidney on right side. A double J-stent was inserted at the left together with a bladder catheter for urinary retention caused by BPH (benign prostate hypertrophy) and levodopa use to maintain GFR at $20 \mathrm{ml} / \mathrm{min} / 1.73 \mathrm{~m}^{2}$. J-stent and bladder catheter changes were mostly complicated and led frequently to traumatic bleeding under the use of clopidogrel and aspirin.

A few weeks ago, a new admission for an aspiration pneumonia complicated by rapid atrial fibrillation and subsequent NSTEMI with new PCI for an occluded old coronary stent was necessary. According to the present guidelines and consensus apixaban $5 \mathrm{mg}$ 2 times daily was added to acetylsalicylic acid $100 \mathrm{mg}$ daily and clopidogrel 75mg daily, establishing modern triple antithrombotic therapy thereby. After a new admission 1 week later for traumatic bleeding following a complicated J-stent change under general anesthesia of 3 hours the patient went home. Restarting full antithrombotic therapy at home led to gross hematuria within 2 days. Following the GP and urologist advice clopidrogrel and Eliquise were stopped and he was advised to drink a lot of water. Urine became blank after 2 days and stayed blank also for 2 days after reinstituting clopidogrel. When Eliquise was taken again massive urinary catheter occurred within 24 hours. All antithrombotic agents were stopped temporarily for a few days and apixaban will not be restarted again.

\section{Discussion}

Acetylsalicylic acid (Aspirin) and clopidogrel (Plavix) are platelet aggregation inhibitors used in the treatment of NSTEMI, unstable angina pectoris, ACS, STEMI/ACS, TIA, PAD (peripheral arterial disease, CVA and atrial fibrillation (A-fib). The risk of hematuria is between $0,1 \%-1,0 \%$. Both prolong bleeding time. Apixaban is a NOAC that inhibits directly factor Xa (FXa) and reduces the generation of thrombin and thereby attenuates its thrombotic effects. 
Bleeding under Eliquis can be reversed by a 2-hour infusion of Andexxa, a recombinant human FXa, when bleeding is life-threatening or doesn't react on stopping apixaban [2].

Although triple therapy with acetylsalicylic acid, clopidogrel and apixaban is tricky with an accumulated risk of bleeding of $15 \%$ this treatment can be indicated and necessary in patients with an ACS and PCIs [3]. Following percutaneous coronary interventions (PCIs) antiplatelet agents are required to prevent-in-stent-thrombosis which has a mortality rate of $50 \%$ to $70 \%$. Additional atrial fibrillation may also require therapy with a NOAC as apixaban [4-6].

\section{Conclusion}

Although the benefits of triple antithrombotic therapy in selected patients seem greater than the bleeding risk, the balance of this cocktail remains very delicate in the elderly. Maintaining this treatment for the advised 6-12 months can be very difficult, especially in elderly patients with urinary catheters or double -J ureter stents or both as this case report shows.

\section{References}

1. Steffel J, Verhamme P, Potpora TS, Albaladejo P, Antz M, et al. (2018) The 2018 European heart rhytm association Practical Guide on the use of non-vitamin $\mathrm{K}$ antagonist oral anticoagulants in patients with atrial fibrillation. Eur Heart J 39(16): 1330-1393.

2. Siegal DM, Curmutte JT, Conolly SJ, Lu G, Conley PB, et al. (2015) Andexanet alfa for the reversal of factor Xa inhibitor activity. N Engl J Med 373: 2413-2424.

3. Sorensen R, Gislason R (2014) Triple antithrombotic therapy: Risky but sometimes necessary. Rev Esp Cardiol 67: 171-175.

4. Janardan J, Gibbs H (2018) Combining anticoagulation and antiplatelet drugs in coronary artery disease. Austr Prescr 41(4): 111-115.

5. Beneivenga L, Komici K, Gorbi G, Cittadini A, Ferrara N, et al. (2018) The management of combined antithrombotic therapy in patients with atrial fibrillation undergoing percutaneous coronary intervention: A particularly complex challenge especially in the elderly. Front Physiol 9: 876.

6. O Riordan M (2018) New consensus statement: NOAC and clopidogrel for most A-fib patients treated with PCI. tctMD/The Heartbeat.
Creative Commons Attribution 4.0

International License

For possible submissions Click Here
Submit Article

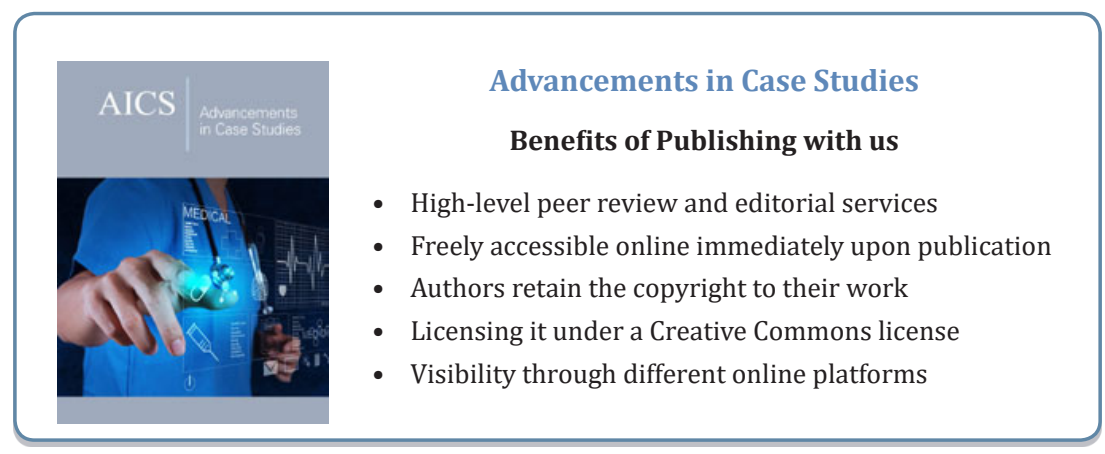

